\title{
SUBORDINATIONS BY ALPHA-CONVEX FUNCTIONS
}

\author{
TeOdOR BulboACĂ
}

\begin{abstract}
Let $H(\mathrm{U})$ be the space of analytic functions in the unit disk $\mathrm{U}$ and let $\mathscr{D}=$ $\{\varphi \in H(\mathrm{U}): \varphi(0)=1, \varphi(z) \neq 0, z \in \mathrm{U}\}$. For the functions $\phi, \varphi \in \mathscr{D}$ we will determine simple sufficient conditions such that

$$
\left[\frac{\varphi(z)}{\phi(z)+(1 / \gamma) z \phi^{\prime}(z)}\right]^{1 / \beta} f(z) \prec k(z) \Rightarrow \mathrm{I}_{\phi, \varphi ; \beta, \gamma}[f](z) \prec k(z),
$$

for all $k \in \mathscr{M}_{1 / \beta}^{\prime}$, where

$$
\mathrm{I}_{\phi, \varphi ; \beta, \gamma}[f](z)=\left[\frac{\gamma}{z^{\gamma} \phi(z)} \int_{0}^{z} f^{\beta}(t) t^{\gamma-1} \varphi(t) \mathrm{d} t\right]^{1 / \beta}
$$

and $\mathscr{M}_{1 / \beta}^{\prime}$ represents the class of $1 / \beta$-convex functions (not necessarily normalized).

In particular, we will give sufficient conditions on $\phi$ and $\varphi$ so that the operators $\mathrm{I}_{\phi, \varphi ; \beta, \gamma}$ are averaging operators on certain subsets of $H(\mathrm{U})$. In addition, some particular cases of the main result, obtained for appropriate choices of the $\phi$ and $\varphi$ functions, will also be given.
\end{abstract}

\section{Introduction}

Let $H(\mathrm{U})$ be the space of analytic functions in the unit disk $\mathrm{U}=\{z \in \boldsymbol{C}$ : $|z|<1\}$ and let $\mathscr{D}=\{\varphi \in H(\mathrm{U}): \varphi(0)=1, \varphi(z) \neq 0, z \in \mathrm{U}\}$. We denote by $\mathscr{A}$ the class of analytic functions in $U$ and usually normalized, i.e.

$$
\mathscr{A}=\left\{f \in H(\mathrm{U}): f(0)=0, f^{\prime}(0)=1\right\} .
$$

If $\beta, \gamma \in C$ with $\beta \neq 0$, for the functions $\phi, \varphi \in \mathscr{D}$ we will define the integral operator $\mathrm{I}_{\phi, \varphi ; \beta, \gamma}: \mathscr{K}_{\varphi ; \beta, \gamma} \rightarrow H(\mathrm{U})$ of the form

$$
\mathrm{I}_{\phi, \varphi ; \beta, \gamma}[f](z)=\left[\frac{\gamma}{z^{\gamma} \phi(z)} \int_{0}^{z} f^{\beta}(t) t^{\gamma-1} \varphi(t) \mathrm{d} t\right]^{1 / \beta},
$$

where $\mathscr{K}_{\varphi ; \beta, \gamma} \subset H(\mathrm{U})$ will be determined in Lemma 3.1, such that the integral operator (1.1) is well defined.

1991 Mathematics Subject Classification: Primary 30C80; Secondary 30C25, 30C45.

Key words and phrases: Differential subordination, averaging operator, subordination chain, univalent function, alpha-convex function.

Received October 22, 2002; revised February 19, 2003. 
The first major result concerning this operator was given in [8], and some particular cases were previously studied in a large number of papers.

For a set $E \subset C$ let denote by co $E$ the convex hull of $E$. In [10] and [4] the authors introduced the concept of averaging (or mean-value) operator on an arbitrary set $K \subset H(\mathrm{U})$, like an operator $\mathrm{I}: K \rightarrow H(\mathrm{U})$ that satisfies

$$
\mathrm{I}[f](0)=f(0) \quad \text { and } \quad \mathrm{I}[f](\mathrm{U}) \subset \operatorname{co} f(\mathrm{U}), \quad \text { for all } f \in K .
$$

For $f, g \in H(\mathrm{U})$ we say that the function $f$ is subordinate to $g$, written $f(z) \prec g(z)$, if $g$ is univalent in $\mathrm{U}, f(0)=g(0)$ and $f(\mathrm{U}) \subseteq g(\mathrm{U})$.

By using several results involving differential subordinations and subordination chains techniques, we will determine simple sufficient conditions on $\phi, \varphi \in \mathscr{D}$ such that

$$
\left[\frac{\varphi(z)}{\phi(z)+(1 / \gamma) z \phi^{\prime}(z)}\right]^{1 / \beta} f(z) \prec k(z) \Rightarrow \mathrm{I}_{\phi, \varphi ; \beta, \gamma}[f](z) \prec k(z),
$$

for all $k \in \mathscr{M}_{1 / \beta}^{\prime}$, where $\mathscr{M}_{1 / \beta}^{\prime}$ represents the class of $1 / \beta$-convex functions (not necessarily normalized) and is given by (2.2). In particular, we will give conditions on $\phi$ and $\varphi$ so that the operators $\mathrm{I}_{\phi, \varphi ; \beta, \gamma}$ are averaging operators on certain subsets of $H(\mathrm{U})$, and in addition, some special cases of the main result obtained for appropriate choices of the $\phi$ and $\varphi$ functions will also be presented.

\section{Preliminaries}

In order to prove our main results, we will need the following definitions and lemmas presented in this section.

Let denote by 2 the set of functions $q$ that are analytic and injective on $\overline{\mathrm{U}} \backslash E(q)$, where

$$
E(q)=\left\{\zeta \in \partial \mathbf{U}: \lim _{z \rightarrow \zeta} q(z)=\infty\right\},
$$

and such that $q^{\prime}(\zeta) \neq 0$ for $\zeta \in \partial \mathrm{U} \backslash E(q)$.

LEMmA $2.1[1]$. Let $q \in \mathscr{Q}$, with $q(0)=a$, and let $p(z)=a+a_{n} z^{n}+\cdots$ be analytic in $\mathrm{U}$ with $p(z) \not \equiv a$ and $n \geq 1$. If $p$ is not subordinate to $q$, then there exist points $z_{0} \in \mathrm{U}$ and $\zeta_{0} \in \partial \mathrm{U} \backslash E(q)$, and an $m \geq n \geq 1$ for which $p\left(|z|<\left|z_{0}\right|\right) \subset$ $q(\mathrm{U})$, and

(i) $\quad p\left(z_{0}\right)=q\left(\zeta_{0}\right)$,

(ii) $z_{0} p^{\prime}\left(z_{0}\right)=m \zeta_{0} q^{\prime}\left(\zeta_{0}\right)$,

(iii) $\operatorname{Re} \frac{z_{0} p^{\prime \prime}\left(z_{0}\right)}{p^{\prime}\left(z_{0}\right)}+1 \geq m \operatorname{Re}\left[\frac{z_{0} q^{\prime \prime}\left(z_{0}\right)}{q^{\prime}\left(z_{0}\right)}+1\right]$.

For $\alpha \in \boldsymbol{R}$, a function $f \in H(\mathrm{U})$ with $f(0)=0$ and $f^{\prime}(0) \neq 0$ is called to be an $\alpha$-convex function (not necessarily normalized), if 


$$
\operatorname{Re}\left[(1-\alpha) \frac{z f^{\prime}(z)}{f(z)}+\alpha\left(\frac{z f^{\prime \prime}(z)}{f^{\prime}(z)}+1\right)\right]>0, \quad z \in \mathrm{U},
$$

and we denote this class by $\mathscr{M}_{\alpha}$. The class of $\alpha$-convex functions was introduced by P. T. Mocanu in [9]. Note that all $\alpha$-convex functions are univalent and starlike, and moreover [7],

$$
\mathscr{M}_{\alpha} \subset \mathscr{M}_{\beta} \subset \mathscr{M}_{0}, \text { for } 0 \leq \frac{\beta}{\alpha} \leq 1 .
$$

For $\alpha \in \boldsymbol{R}$ we denote by

$$
\mathscr{M}_{\alpha}^{\prime}=\left\{f \in H(\mathrm{U}): f^{\prime}(0) \neq 0, \operatorname{Re}\left[(1-\alpha) \frac{z f^{\prime}(z)}{f(z)}+\alpha\left(\frac{z f^{\prime \prime}(z)}{f^{\prime}(z)}+1\right)\right]>0, z \in \mathrm{U}\right\},
$$

and then

$$
\mathscr{K}^{\prime} \equiv \mathscr{M}_{1}^{\prime}=\left\{f \in H(\mathrm{U}): f^{\prime}(0) \neq 0, \operatorname{Re} \frac{z f^{\prime \prime}(z)}{f^{\prime}(z)}+1>0, z \in \mathrm{U}\right\}
$$

represents the class of convex functions (not necessarily normalized) in U.

The next lemma gives us a necessary and sufficient condition for an operator to be an averaging operator.

Lemma 2.2 [10], [4, Lemma 2]. Let $K \subset H(\mathrm{U})$ and let an operator $\mathrm{I}: K \rightarrow H(\mathrm{U})$ that satisfies $\mathrm{I}[f](0)=f(0)$ for all $f \in K . \quad A$ necessary and sufficient condition for $\mathrm{I}$ to be an averaging operator on $K$ is that

$$
f \in K, k \text { convex and } f(z) \prec k(z) \Rightarrow \mathrm{I}[f](z) \prec k(z) .
$$

Let $c \in C$ with $\operatorname{Re} c>0$, and let $N=N(c)=(|c| \sqrt{1+2 \operatorname{Re} c}+\operatorname{Im} c) / \operatorname{Re} c$. If $\chi$ is the univalent function $\chi(z)=2 N z /\left(1-z^{2}\right)$, then we define the open door function $R_{c}$ by

$$
R_{c}(z)=\chi\left(\frac{z+b}{1+\bar{b} z}\right), \quad z \in U
$$

where $b=\chi^{-1}(c)$.

Remark that $R_{c}$ is univalent in $\mathrm{U}, R_{c}(0)=c$ and $R_{c}(U)=\chi(U)$ is the complex plane slit along the half-lines $\operatorname{Re} w=0, \operatorname{Im} w \geq N$ and $\operatorname{Re} w=0$, $\operatorname{Im} w \leq-N$.

Lemma $2.3[3$, Theorem 1]. Let $\phi, \varphi \in \mathscr{D}$ and let $\alpha, \beta, \gamma$ and $\delta$ be complex numbers with $\beta \neq 0, \alpha+\delta=\beta+\gamma$ and $\operatorname{Re}(\alpha+\delta)>0$. If $f \in \mathscr{A}$ satisfies

$$
\alpha \frac{z f^{\prime}(z)}{f(z)}+\frac{z \varphi^{\prime}(z)}{\varphi(z)}+\delta \prec R_{\alpha+\delta}(z)
$$

and the function $F$ is defined by 


$$
F(z)=\left[\frac{\beta+\gamma}{z^{\gamma} \phi(z)} \int_{0}^{z} f^{\alpha}(t) t^{\delta-1} \varphi(t) \mathrm{d} t\right]^{1 \beta}=z+\cdots,
$$

then $F \in \mathscr{A}, F(z) / z \neq 0, \forall z \in \mathrm{U}$ and

$$
\operatorname{Re}\left[\beta \frac{z F^{\prime}(z)}{F(z)}+\frac{z \phi^{\prime}(z)}{\phi(z)}+\gamma\right]>0, \quad z \in \mathrm{U} .
$$

(All powers in (2.4) are principal ones.)

Lemma 2.4 [6, Lemma 1.2c]. Let $n \geq 0$ be an integer and let $\gamma \in \boldsymbol{C}$, with $\operatorname{Re} \gamma>-n$. If $f(z)=\sum_{m \geq n} a_{m} z^{m}$ is analytic in $\mathrm{U}$ and $F$ is defined by

$$
F(z)=\frac{1}{z^{\gamma}} \int_{0}^{z} f(t) t^{\gamma-1} \mathrm{~d} t,
$$

then $F(z)=\sum_{m \geq n} a_{m} z^{m} /(m+\gamma)$ is analytic in $\mathrm{U}$.

Lemma 2.5 [2, Theorem 2], [4, Theorem 2]. Let $k$ be convex (univalent) in $\mathrm{U}$ and let $A \geq 0$. Suppose $M>4 /\left|h^{\prime}(0)\right|$ and that $B$ and $D$ are analytic in $\mathrm{U}$, with $D(0)=0$ and

$$
\operatorname{Re} B(z) \geq A+M|D(z)|, \quad z \in \mathrm{U} .
$$

If $p$ is analytic in $\mathrm{U}$ with $p(0)=k(0)$, and if $p$ satisfies

$$
A z^{2} p^{\prime \prime}(z)+B(z) z p^{\prime}(z)+p(z)+D(z) \prec k(z),
$$

then $p(z) \prec k(z)$.

A function $L: \mathrm{U} \times[0,+\infty) \rightarrow \boldsymbol{C}$ is called a subordination (or a Loewner) chain if $L(\cdot ; t)$ is analytic and univalent in $\mathrm{U}$ for all $t \geq 0$, and $L(z ; s) \prec L(z ; t)$, when $0 \leq s \leq t$.

Lemma $2.6\left[11\right.$, p. 159]. The function $L(z ; t)=a_{1}(t) z+a_{2}(t) z^{2}+\cdots$, with $a_{1}(t) \neq 0$ for $t \geq 0$, and $\lim _{t \rightarrow \infty}\left|a_{1}(t)\right|=\infty$, is a subordination chain if and only if there exist constants $r \in(0,1]$ and $M>0$ such that

(i) $L(z ; t)$ is analytic in $|z|<r$ for each $t \geq 0$, locally absolutely continuous in $[0, \infty)$ for each $|z|<r$, and satisfies

$$
|L(z ; t)| \leq M\left|a_{1}(t)\right|, \quad \text { for }|z|<r \text { and } t \geq 0
$$

(ii) there exists a function $p(z, t)$ analytic in $\mathrm{U}$ for all $t \in[0, \infty)$ and measurable in $[0, \infty)$ for each $z \in \mathrm{U}$, such that $\operatorname{Re} p(z, t)>0$ for $z \in \mathrm{U}, t \in[0, \infty)$, and

$$
\frac{\partial L(z ; t)}{\partial t}=z \frac{\partial L(z ; t)}{\partial z} p(z, t), \quad \text { for }|z|<r \text { and for almost all } t \in[0, \infty) .
$$

\section{Main results}

First we need to determine sufficient conditions on the $\phi$ and $\varphi$ functions such that the integral operators $\mathbf{I}_{\phi, \varphi ; \beta, \gamma}$ are well defined. 
From the fact that

$$
\mathrm{I}_{\phi, \varphi ; \beta, \gamma}[f](z)=\left(\frac{\gamma}{\beta+\gamma}\right)^{1 / \beta} \tilde{F}(z), \quad \text { if } \gamma \neq 0
$$

where

$$
\tilde{F}(z)=\left[\frac{\beta+\gamma}{z^{\gamma} \phi(z)} \int_{0}^{z} f^{\beta}(t) t^{\gamma-1} \varphi(t) \mathrm{d} t\right]^{1 / \beta},
$$

in order to determine the subset $\mathscr{K}_{\varphi ; \beta, \gamma} \subset H(\mathrm{U})$ so that the operator $\mathrm{I}_{\phi, \varphi ; \beta, \gamma}$ given by (1.1) is well defined, we need to find the set $\mathscr{K}_{\varphi ; \beta, \gamma}$ such that $\tilde{F} \in H(\mathrm{U})$ for all $f \in \mathscr{K}_{\varphi ; \beta, \gamma}$.

Lemma 3.1. Let $\beta, \gamma \in C$ with $\beta \neq 0, \operatorname{Re}(\beta+\gamma)>0$ and let $\phi, \varphi \in \mathscr{D}$. If $R_{\beta+\gamma}$ represents the open door function defined by (2.3) and if

$$
\mathscr{K}_{\varphi ; \beta, \gamma}=\left\{f \in \mathscr{A}: \beta \frac{z f^{\prime}(z)}{f(z)}+\frac{z \varphi^{\prime}(z)}{\varphi(z)}+\gamma \prec R_{\beta+\gamma}(z)\right\}, \quad \text { for } \beta \neq 1,
$$

and

$$
\mathscr{K}_{\varphi ; 1, \gamma}=H(\mathrm{U}), \quad \text { for } \beta=1 \text {, if in addition } \operatorname{Re} \gamma>0,
$$

then the integral operator $\mathrm{I}_{\phi, \varphi ; \beta, \gamma}$ is well-defined.

Proof. If $\beta \neq 1$, from (3.1) by using Lemma 2.3 for the case $\alpha=\beta$ and $\delta=\gamma$ we deduce the first part of the result.

If $\beta=1$, denoting $t=w z$ we have

$$
\tilde{F}(z)=\frac{\gamma+1}{\phi(z)} \int_{0}^{1} f(w z) \varphi(w z) w^{\gamma-1} \mathrm{~d} w
$$

and from (3.1) according to Lemma 2.4 we obtain the second part of our result.

Using Lemma 2.3, the previous result and the relation (3.1), respectively Lemma 2.4 and the relation (3.1) we deduce the next two remarks:

Remark 3.1. Under the assumptions of Lemma 3.1, for $\beta \neq 1$, we have

$$
\tilde{F} \in \mathscr{A}, \quad \frac{\tilde{F}(z)}{z} \neq 0, \quad z \in U \quad \text { and } \quad \operatorname{Re}\left[\beta \frac{z \tilde{F}^{\prime}(z)}{\tilde{F}(z)}+\frac{z \phi^{\prime}(z)}{\phi(z)}+\gamma\right]>0, \quad z \in \mathrm{U},
$$

hence,

$$
\mathrm{I}_{\phi, \varphi ; \beta, \gamma}[f](z)=\left(\frac{\gamma}{\beta+\gamma}\right)^{1 / \beta} z+\cdots \in H(\mathrm{U}), \quad \forall f \in \mathscr{K}_{\varphi ; \beta, \gamma} \text { and } \beta \neq 1 .
$$

Remark 3.2. Under the assumptions of Lemma 3.1, we have

$$
\mathrm{I}_{\phi, \varphi ; \beta, \gamma}[f](0)=f(0), \quad \forall f \in \mathscr{K}_{\varphi ; \beta, \gamma} .
$$



that

TheOREM 3.1. Let $\beta, \gamma \in \boldsymbol{C}$ with $\beta>0$ and $\operatorname{Re} \gamma>0$, let $\phi, \varphi \in \mathscr{D}$ and suppose (i) $\operatorname{Re}\left[\frac{z \phi^{\prime}(z)}{\phi(z)}+\gamma\right]>0, \quad z \in \mathrm{U}$.

Let $k \in \mathscr{M}_{1 / \beta}^{\prime}$ and $f \in \mathscr{K}_{\varphi ; \beta, \gamma}$. Then

$$
\left[\frac{\varphi(z)}{\phi(z)+(1 / \gamma) z \phi^{\prime}(z)}\right]^{1 / \beta} f(z) \prec k(z) \Rightarrow \mathrm{I}_{\phi, \phi ; \beta, \gamma}[f](z) \prec k(z) .
$$

Proof. Since $\beta \neq 0$ and $\operatorname{Re}(\beta+\gamma)>0$, according to Lemma 3.1, the operator $\mathrm{I}_{\phi, \varphi ; \beta, \gamma}$ is well-defined on the set $\mathscr{K}_{\varphi ; \beta, \gamma}$.

From the assumption $\left[\varphi(z) /\left(\phi(z)+(1 / \gamma) z \phi^{\prime}(z)\right)\right]^{1 / \beta} f(z) \prec k(z) \quad$ we have $f(0)=k(0)$. If $\beta \neq 1$ then $f \in \mathscr{A}$, hence $k(0)=f(0)=0$ i.e. $k \in \mathscr{M}_{1 / \beta}$, so it follows that $k$ is univalent in $\mathrm{U}$. If $\beta=1$ then $k \in \mathscr{K}^{\prime} \equiv \mathscr{M}_{1}^{\prime}$, hence $k$ is a convex (and univalent) function in $\mathrm{U}$.

If we denote by $F(z)=\mathrm{I}_{\phi, \varphi ; \beta, \gamma}[f](z)$, then by Remark 3.2 we have $F(0)=$ $f(0)$ and

$$
\left[\frac{\varphi(z)}{\phi(z)+(1 / \gamma) z \phi^{\prime}(z)}\right]^{1 / \beta} f(z)=F(z)\left[\frac{\beta}{\gamma} \frac{z F^{\prime}(z)}{F(z)} \frac{1}{H(z)}+1\right]^{1 / \beta}
$$

where

$$
H(z)=1+\frac{1}{\gamma} \frac{z \phi^{\prime}(z)}{\phi(z)} .
$$

Remark that the assumption (i) implies $H(z) \neq 0$ for all $z \in \mathrm{U}$.

Thus, we need to prove the next implication:

$$
F(z)\left[\frac{\beta}{\gamma} \frac{z F^{\prime}(z)}{F(z)} \frac{1}{H(z)}+1\right]^{1 / \beta} \prec k(z) \Rightarrow F(z) \prec k(z) .
$$

For the particular case $\beta=1$, the implication (3.3) becomes

$$
F(z)+\frac{1}{\gamma H(z)} z F^{\prime}(z) \prec k(z) \Rightarrow F(z) \prec k(z) .
$$

According to Lemma 2.5 for $A=0$ and $D(z) \equiv 0$, and by using the inequality (i) we deduce that the above implication holds.

Now we will prove our result for the case $\beta \neq 1$. Without loss of generality we can assume that $k$ satisfies the conditions of the theorem on the closed disk $\overline{\mathrm{U}}$ and $k^{\prime}(\zeta) \neq 0$ for $|\zeta|=1$. If not, then we replace $f, k, \phi$ and $\varphi$ with $f_{r}(z)=f(r z), k_{r}(z)=k(r z), \phi_{r}(z)=\phi(r z)$ and $\varphi_{r}(z)=\varphi(r z)$ where $0<r<1$, and then $k_{r}$ is univalent on $\overline{\mathrm{U}}$. Since

$$
\left[\frac{\varphi_{r}(z)}{\phi_{r}(z)+(1 / \gamma) z \phi_{r}^{\prime}(z)}\right]^{1 / \beta} f_{r}(z) \prec k_{r}(z),
$$


we would then prove that

$$
F_{r}(z)=F(r z)=\mathrm{I}_{\phi_{r}, \varphi_{r} ; \beta, \gamma}\left[f_{r}\right](z) \prec k_{r}(z), \quad \text { for } 0<r<1,
$$

and by letting $r \rightarrow 1^{-}$we obtain $F(z) \prec k(z)$.

If we suppose that the implication (3.3) is not true, i.e. $F(z) \nless k(z)$, then from Lemma 2.1 there exist points $z_{0} \in \mathrm{U}$ and $\zeta_{0} \in \partial \mathbf{U}$, and a number $m \geq 1$, such that

$$
F\left(z_{0}\right)=k\left(\zeta_{0}\right)
$$

and

$$
z_{0} F^{\prime}\left(z_{0}\right)=m \zeta_{0} k^{\prime}\left(\zeta_{0}\right)
$$

To prove the implication (3.3) we define the function $L: \mathrm{U} \times[0, \infty) \rightarrow \boldsymbol{C}$ by

$$
L(z ; t)=k(z)\left[\frac{\beta}{\gamma} t \frac{z k^{\prime}(z)}{k(z)} \frac{1}{H\left(z_{0}\right)}+1\right]^{1 / \beta}=a_{1}(t) z+\cdots,
$$

and we will show that $L(z ; t)$ is a subordination chain. have

From the fact that $z k^{\prime}(z) /\left.k(z)\right|_{z=0}=1$ and the assumptions (i) and $\beta>0$, we

$$
\left.\operatorname{Re} \frac{\beta}{\gamma} \frac{z k^{\prime}(z)}{k(z)}\right|_{z=0} \frac{1}{H\left(z_{0}\right)}>0
$$

hence $L(z ; t)$ is analytic in $|z|<r<1$, for sufficient small $r>0$ and for all $t \geq 0$. We also have that $L(z ; t)$ is continuously differentiable on $[0, \infty)$ for each $|z|<r<1$.

A simple calculus shows that

$$
a_{1}(t)=\frac{\partial L(0 ; t)}{\partial z}=k^{\prime}(0)\left[\frac{\beta}{\gamma} t \frac{1}{H\left(z_{0}\right)}+1\right]^{1 / \beta},
$$

and because $k^{\prime}(0) \neq 0$, from (i) and $\beta>0$ we deduce

$$
\operatorname{Re}\left[\frac{\beta}{\gamma} t \frac{1}{H\left(z_{0}\right)}+1\right] \geq 1>0, \quad \forall t \geq 0,
$$

hence $a_{1}(t) \neq 0, \forall t \geq 0$. From (i) we have $(\beta / \gamma) t\left(1 / H\left(z_{0}\right)\right) \neq 0, \forall z_{0} \in \mathrm{U}$ and $\forall t>0$, so we obtain that $\lim _{t \rightarrow \infty}\left|a_{1}(t)\right|=\infty$.

Using the definition (3.6), by a directly computation we obtain

$$
\operatorname{Re}\left[z \frac{\partial L / \partial z}{\partial L / \partial t}\right]=t \beta \operatorname{Re}\left[\left(1-\frac{1}{\beta}\right) \frac{z k^{\prime}(z)}{k(z)}+\frac{1}{\beta}\left(1+\frac{z k^{\prime \prime}(z)}{k^{\prime}(z)}\right)\right]+\operatorname{Re}\left[\gamma H\left(z_{0}\right)\right] .
$$

From the above relation, by using the fact that $k \in \mathscr{M}_{1 / \beta}^{\prime}$ and the assumption (i), we deduce that

$$
\operatorname{Re}\left[z \frac{\partial L / \partial z}{\partial L / \partial t}\right]>0, \quad \forall z \in \mathrm{U}, \forall t \geq 0
$$


and according to Lemma 2.6 we conclude that $L(z ; t)$ is a subordination chain. This implies in particular:

$$
k(z)=L(z ; 0) \prec L(z ; t), \quad \forall t \geq 0 .
$$

Using the equality (3.2) and the relations (3.4) and (3.5) we obtain

$$
\begin{aligned}
{\left[\frac{\varphi\left(z_{0}\right)}{\phi\left(z_{0}\right)+(1 / \gamma) z_{0} \phi^{\prime}\left(z_{0}\right)}\right]^{1 / \beta} f\left(z_{0}\right) } & =F\left(z_{0}\right)\left[\frac{\beta}{\gamma} \frac{z_{0} F^{\prime}\left(z_{0}\right)}{F\left(z_{0}\right)} \frac{1}{H\left(z_{0}\right)}+1\right]^{1 / \beta} \\
& =k\left(\zeta_{0}\right)\left[\frac{\beta}{\gamma} m \frac{\zeta_{0} k^{\prime}\left(\zeta_{0}\right)}{k\left(\zeta_{0}\right)} \frac{1}{H\left(z_{0}\right)}+1\right]^{1 / \beta} \\
& =L\left(\zeta_{0} ; m\right), \quad m \geq 1,
\end{aligned}
$$

and then, according to (3.7) we deduce that

$$
\left[\frac{\varphi\left(z_{0}\right)}{\phi\left(z_{0}\right)+(1 / \gamma) z_{0} \phi^{\prime}\left(z_{0}\right)}\right]^{1 / \beta} f\left(z_{0}\right)=L\left(\zeta_{0} ; m\right) \notin k(\mathrm{U}) .
$$

This last relation contradicts the assumption $\left[\varphi(z) /\left(\phi(z)+(1 / \gamma) z \phi^{\prime}(z)\right)\right]^{1 / \beta} f(z) \prec$ $k(z)$, then we finally conclude that $F(z) \prec k(z)$.

\section{Particular cases}

In this section we will discuss several particular cases of Theorem $3.1 \mathrm{ob}-$ tained for appropriate choices of the $\phi$ and $\varphi$ functions.

1. For a given function $\phi \in \mathscr{D}$, taking $\varphi(z)=\phi(z)+(1 / \gamma) z \phi^{\prime}(z)$ in Theorem 3.1, then $\varphi(0)=1$ and the assumption (i) of the theorem is equivalent to

$$
\operatorname{Re}\left[\gamma \frac{\varphi(z)}{\phi(z)}\right]>0, \quad z \in \mathrm{U}
$$

Since $\phi(z) \neq 0, \forall z \in \mathrm{U}$, from the above inequality it follows that $\varphi \in \mathscr{D}$ and then we obtain: that

Corollary 4.1. Let $\beta, \gamma \in C$ with $\beta>0$ and $\operatorname{Re} \gamma>0$, let $\phi \in \mathscr{D}$ and suppose

$$
\text { (i) } \operatorname{Re}\left[\frac{z \phi^{\prime}(z)}{\phi(z)}+\gamma\right]>0, \quad z \in \mathrm{U}
$$

Let $k \in \mathscr{M}_{1 / \beta}^{\prime}$ and $f \in \mathscr{K}_{\phi+(1 / \gamma) z \phi^{\prime} ; \beta, \gamma}$. Then

$$
f(z) \prec k(z) \Rightarrow \mathrm{I}_{\phi, \phi+(1 / \gamma) z \phi^{\prime} ; \beta, \gamma}[f](z) \prec k(z),
$$

where

$$
\mathrm{I}_{\phi, \phi+(1 / \gamma) z \phi^{\prime} ; \beta, \gamma}[f](z)=\left[\frac{\gamma}{z^{\gamma} \phi(z)} \int_{0}^{z} f^{\beta}(t) t^{\gamma-1}\left(\phi(t)+\frac{1}{\gamma} t \phi^{\prime}(t)\right) \mathrm{d} t\right]^{1 / \beta} .
$$


Using this corollary in the special case $k(z)=f(z)$ we obtain the next example:

Example 4.1. Let $\beta, \gamma \in C$ with $\beta>0$ and $\operatorname{Re} \gamma>0$, let $\phi \in \mathscr{D}$ and suppose that

$$
\text { (i) } \operatorname{Re}\left[\frac{z \phi^{\prime}(z)}{\phi(z)}+\gamma\right]>0, \quad z \in \mathrm{U}
$$

If $f \in \mathscr{K}_{\phi+(1 / \gamma) z \phi^{\prime} ; \beta, \gamma} \cap \mathscr{M}_{1 / \beta}^{\prime}$, then

$$
\mathrm{I}_{\phi, \phi+(1 / \gamma) z \phi^{\prime} ; \beta, \gamma}[f](z) \prec f(z),
$$

where $\mathrm{I}_{\phi, \phi+(1 / \gamma) \not \phi^{\prime} ; \beta, \gamma}[f]$ is given by $(4.1)$.

Our next result deals with a general class of averaging integral operators. that

Corollary 4.2. Let $\beta, \gamma \in C$ with $\beta \geq 1$ and $\operatorname{Re} \gamma>0$, let $\phi \in \mathscr{D}$ and suppose

$$
\text { (i) } \operatorname{Re}\left[\frac{z \phi^{\prime}(z)}{\phi(z)}+\gamma\right]>0, \quad z \in \mathrm{U} \text {. }
$$

Then the integral operator $\mathrm{I}_{\phi, \phi+(1 / \gamma) z \phi^{\prime} ; \beta, \gamma}$ given by (4.1) is an averaging operator on $\mathscr{K}_{\phi+(1 / \gamma) z \phi^{\prime} ; \beta, \gamma}$.

Proof. If $f \in \mathscr{K}_{\phi+(1 / \gamma) z \phi^{\prime} ; \beta, \gamma}$, then from Remark 3.2 we have $\mathbf{I}_{\phi, \phi+(1 / \gamma) z \phi^{\prime} ; \beta, \gamma}$ $[f](0)=f(0)$. Let consider an arbitrary convex function $k$ such that $f(z) \prec$ $k(z)$.

For the case $\beta=1$ we have $k \in \mathscr{K}^{\prime} \equiv \mathscr{M}_{1}^{\prime}$ and, according to Corollary 4.1 we deduce that $\mathrm{I}_{\phi, \phi+(1 / \gamma) z \phi^{\prime} ; 1, \gamma}[f](z) \prec k(z)$.

For the case $\beta>1$, since $f(z) \prec k(z)$ and $f \in \mathscr{K}_{\phi+(1 / \gamma) z \phi^{\prime} ; \beta, \gamma}$ then $k(0)=$ $f(0)=0$. From (2.1) we have $k \in \mathscr{M}_{1} \subset \mathscr{M}_{1 / \beta} \subset \mathscr{M}_{1 / \beta}^{\prime}$ for $\beta>1$, and using Corollary 4.1 we obtain that $\mathrm{I}_{\phi, \phi+(1 / \gamma) z \phi^{\prime} ; \beta, \gamma}[f](z) \prec k(z)$ for $\beta>1$.

Now, from Lemma 2.2 we conclude that in the both two cases the integral operator $\mathbf{I}_{\phi, \phi+(1 / \gamma) z \phi^{\prime} ; \beta, \gamma}$ is an averaging operator on $\mathscr{K}_{\phi+(1 / \gamma) z \phi^{\prime} ; \beta, \gamma}$.

Remark 4.1. Remark that this corollary generalizes Theorem 1 of [5] that may be obtained for the particular case $\phi(z)=1$.

2. Taking $\phi(z)=\varphi(z)=e^{\lambda z}$ with $\lambda \in \boldsymbol{C}$ in Theorem 3.1, then $\phi \equiv \varphi \in \mathscr{D}$ and the condition (i) of the theorem reduces to $\operatorname{Re}(\lambda z+\gamma)>0, \forall z \in \mathrm{U}$. Since this inequality holds whenever $\operatorname{Re} \gamma \geq|\lambda|$, we obtain:

Corollary 4.3. Let $\beta, \gamma \in C$ with $\beta>0$ and $\operatorname{Re} \gamma>0$. For a number $\lambda \in C$, suppose in addition that $\operatorname{Re} \gamma \geq|\lambda|$. Let $k \in \mathscr{M}_{1 / \beta}^{\prime}$ and $f \in \mathscr{K}_{e^{\lambda z} ; \beta, \gamma}$. Then 


$$
\left(\frac{\gamma}{\lambda z+\gamma}\right)^{1 / \beta} f(z) \prec k(z) \Rightarrow \mathrm{I}_{e^{\lambda z}, e^{\lambda z} ; \beta, \gamma}[f](z) \prec k(z),
$$

where

$$
\mathrm{I}_{e^{\lambda z}, e^{\lambda z} ; \beta, \gamma}[f](z)=\left[\frac{\gamma}{z^{\gamma} e^{\lambda z}} \int_{0}^{z} f^{\beta}(t) t^{\gamma-1} e^{\lambda t} \mathrm{~d} t\right]^{1 / \beta} .
$$

By the same reasons, if we take $\phi(z)=\varphi(z)=e^{\lambda z}$ with $\lambda \in \boldsymbol{C}$ in Corollary 4.2, we have:

Corollary 4.4. Let $\beta, \gamma \in C$ with $\beta>0$ and $\operatorname{Re} \gamma>0$. For a number $\lambda \in C$, suppose in addition that $\operatorname{Re} \gamma \geq|\lambda|$. Then the integral operator $\mathrm{I}_{e^{\lambda z}, e^{\lambda z} ; \beta, \gamma}$ given by

$$
\mathrm{I}_{e^{\lambda z}, e^{\lambda z}+(\lambda z / \gamma) e^{\lambda z} ; \beta, \gamma}[f](z)=\left[\frac{\gamma}{z^{\gamma} e^{\lambda z}} \int_{0}^{z} f^{\beta}(t) t^{\gamma-1}\left(e^{\lambda z}+\frac{\lambda z}{\gamma} e^{\lambda z}\right) \mathrm{d} t\right]^{1 / \beta}
$$

is an averaging operator on $\mathscr{K}_{e^{i z} ; \beta, \gamma}$.

Remark 4.2. Note that this corollary also extends Theorem 1 of [5] that may be obtained for the special case $\lambda=0$.

3. Considering $\phi(z)=1+\lambda z, \lambda \in \boldsymbol{C}$, then $\phi(0)=1$ and for $|\lambda| \leq 1$ we have $\phi(z) \neq 0, \forall z \in \mathrm{U}$, i.e. $\phi \in \mathscr{D}$.

It is easy to check that the condition (i) of Theorem 3.1 becomes

$$
\operatorname{Re}\left[\frac{\lambda z}{1+\lambda z}+\gamma\right]>0, \quad z \in \mathrm{U}
$$

Letting $\chi(\zeta)=\zeta /(1+\zeta)$, since $\chi^{\prime}(0) \neq 0$ and

$$
\operatorname{Re} \frac{\zeta \chi^{\prime \prime}(\zeta)}{\chi^{\prime}(\zeta)}+1=\operatorname{Re} \frac{1-\zeta}{1+\zeta}>0, \quad|\zeta|<1,
$$

the function $\chi$ is a convex function in $D=\{\zeta \in C:|\zeta|<|\lambda|\}$, if $|\lambda| \leq 1$. From the fact that $\chi(\bar{\zeta})=\bar{\chi}(\zeta), \zeta \in D$, we deduce that the function $\chi$ maps the disk $D$ onto the convex domain $\chi(D)$ that is symmetric with respect the real axis. Hence

$$
\inf \left\{\frac{\lambda z}{1+\lambda z}: z \in \mathrm{U}\right\}=t(-|\lambda|)=\frac{|\lambda|}{|\lambda|-1}, \text { for }|\lambda|<1,
$$

which shows that the condition (4.2) holds whenever $\operatorname{Re} \gamma \geq|\lambda| /(1-|\lambda|)$, for $|\lambda|<1$. From here, by using Theorem 3.1 we have:

Corollary 4.5. Let $\beta, \gamma \in C$ with $\beta>0$ and $\operatorname{Re} \gamma>0$. Let $\varphi \in \mathscr{D}$ and for a number $\lambda \in \boldsymbol{C}$ with $|\lambda|<1$, suppose in addition that 


$$
\operatorname{Re} \gamma \geq \frac{|\lambda|}{1-|\lambda|} .
$$

Let $k \in \mathscr{M}_{1 / \beta}^{\prime}$ and $f \in \mathscr{K}_{\varphi ; \beta, \gamma}$. Then

$$
\left[\frac{\varphi(z)}{1+((\gamma+1) / \gamma) \lambda z}\right]^{1 / \beta} f(z) \prec k(z) \Rightarrow \mathrm{I}_{1+\lambda z, \varphi ; \beta, \gamma}[f](z) \prec k(z) .
$$

we have:

By the same reasons, if we take $\phi(z)=1+\lambda z$ with $|\lambda|<1$ in Corollary 4.2,

Corollary 4.6. Let $\beta, \gamma \in \boldsymbol{C}$ with $\beta>0$ and $\operatorname{Re} \gamma>0$. For a number $\lambda \in \boldsymbol{C}$ with $|\lambda|<1$, suppose in addition that

$$
\operatorname{Re} \gamma \geq \frac{|\lambda|}{1-|\lambda|} .
$$

Then the integral operator $\mathrm{I}_{1+\lambda z, 1+((\gamma+1) / \gamma) z z ; \beta, \gamma}$ given by

$$
\mathrm{I}_{1+\lambda z, 1+((\gamma+1) / \gamma) \lambda z ; \beta, \gamma}[f](z)=\left[\frac{\gamma}{z^{\gamma}(1+\lambda z)} \int_{0}^{z} f^{\beta}(t) t^{\gamma-1}\left(1+\frac{\gamma+1}{\gamma} \lambda t\right) \mathrm{d} t\right]^{1 / \beta}
$$

is an averaging operator on $\mathscr{K}_{1+((\gamma+1) / \gamma) \lambda z ; \beta, \gamma}$.

Remark 4.3. This corollary also extends Theorem 1 of [5] that can be obtained for the particular case $\lambda=0$.

4. If we take $\phi(z)=(1+z)^{2 a}$ with $a \leq 0$ in Theorem 3.1, then the condition (i) of the theorem reduces to

$$
\operatorname{Re}\left[\frac{2 a z}{1+z}+\gamma\right]>0, \quad z \in \mathrm{U}
$$

If $a<0$, the function $\chi(z)=2 a z /(1+z)$ maps the unit disk $\mathrm{U}$ onto the halfplane $\Delta=\{w \in C: \operatorname{Re} w>a\}$, and we deduce that the above inequality holds if and only if $\operatorname{Re} \gamma \geq-a$.

If $a=0$, the same inequality holds for all $\gamma \in C$ with $\operatorname{Re} \gamma>0$, hence we obtain the next result:

Corollary 4.7. Let $\beta, \gamma \in C$ with $\beta>0$ and $\operatorname{Re} \gamma>0$. Let $\varphi \in \mathscr{D}$ and for a number $a \leq 0$ suppose in addition that

$$
\operatorname{Re} \gamma \geq-a \text {. }
$$

Let $k \in \mathscr{M}_{1 / \beta}^{\prime}$ and $f \in \mathscr{K}_{\varphi ; \beta, \gamma}$. Then

$$
\left[\frac{\varphi(z)}{(1+z)^{2 a-1}(1+((2 a+\gamma) / \gamma) z)}\right]^{1 / \beta} f(z) \prec k(z) \Rightarrow \mathrm{I}_{(1+z)^{2 a}, \varphi ; \beta, \gamma}[f](z) \prec k(z) .
$$


Similarly, by taking $\phi(z)=(1+z)^{2 a}$ with $a \leq 0$ in Corollary 4.2 we have:

Corollary 4.8. Let $\beta, \gamma \in \boldsymbol{C}$ with $\beta>0$ and $\operatorname{Re} \gamma>0$. For a number $a \leq 0$ suppose in addition that

$$
\operatorname{Re} \gamma \geq-a \text {. }
$$

Then the integral operator $\mathrm{I}_{(1+z)^{2 a},(1+z)^{2 a-1}(1+((2 a+\gamma) / \gamma) z) ; \beta, \gamma}$ given by

$$
\begin{aligned}
& \mathrm{I}_{(1+z)^{2 a},(1+z)^{2 a-1}(1+((2 a+\gamma) / \gamma) z) ; \beta, \gamma}[f](z) \\
& \quad=\left[\frac{\gamma}{z^{\gamma}(1+z)^{2 a}} \int_{0}^{z} f^{\beta}(t) t^{\gamma-1}(1+t)^{2 a-1}\left(1+\frac{2 a+\gamma}{\gamma} t\right) \mathrm{d} t\right]^{1 / \beta}
\end{aligned}
$$

is an averaging operator on $\mathscr{K}_{(1+z)^{2 a-1}(1+((2 a+\gamma) / \gamma) z) ; \beta, \gamma}$.

Remark 4.4. For the particular case $a=0$ we remark that this corollary represents Theorem 1 of [5].

\section{REFERENCES}

[1] S. S. Miller and P. T. Mocanu, Differential subordinations and univalent functions, Michigan Math. J., 28 (1981), 157-171.

[2] —, Mean-value theorems in the complex plane, Babeş-Bolyai Univ. Fac. of Math. Res. Sem., Seminar on Geometric Function Theory, Preprint 5 (1986), 63-67.

[ 3 ] — Classes of univalent integral operators, J. Math. Anal. Appl., 157, 1 (1991), 147-167.

[ 4 ] - Averaging operators and generalized Robinson differential inequality, J. Math. Anal. Appl., 13, 2 (1993), 459-469.

[ 5 ] — A class of nonlinear averaging operators, J. Math. Anal. Appl., 197 (1996), 318-323.

[6] —, Differential Subordinations. Theory and Applications, Marcel Dekker, New York, 1999.

[7] AND M. O. READE, Bazilevič functions and generalized convexity, Rev. Roumaine Math. Pures Appl., 19 (1974), 213-224.

[8] —, Starlike integral operators, Pacific J. Math., 79 (1978), 157-168.

[9] P. T. Mocanu, Une propriété de conexité generalisée dans la théorie de la representation conforme, Mathematica (Cluj), 11, 34 (1969), 127-133.

[10] - Second order averaging operators for analytic functions, Rev. Roumaine Math. Pures Appl., 33, 10 (1988), 875-881.

[11] Ch. Pommerenke, Univalent Functions, Vanderhoeck and Ruprecht, Göttingen, 1975.

DEPARTMENT OF FunCTION THEORY

Faculty of Mathematics and Computer Science, Babeş-Bolyai University 3400 Cluj-Napoca, Romania

E-mail: bulboaca@math.ubbcluj.ro 